\title{
Metocean Comparisons of Jason-2 and AltiKa - A method to develop a new wind speed algorithm
}

\author{
Graham D. Quartly \\ Plymouth Marine Laboratory (e: gqu@pml.ac.uk) \\ This is an Accepted Manuscript of an article published by Taylor \& Francis, to appear in Marine Geodesy \\ SARAL/AltiKa special issue. doi: 10.1080/01490419.2014.988834
}

\begin{abstract}
As well as range, the AltiKa altimeter provides estimates of wave height, $\mathrm{H}_{\mathrm{s}}$ and normalized backscatter, $\sigma^{0}$, that need to be assessed prior to statistics based on them being included in climate databases. An analysis of crossovers with the Jason-2 altimeter shows AltiKa $\mathrm{H}_{\mathrm{s}}$ values to be biased high by only $\sim 0.05 \mathrm{~m}$, with a standard deviation (s.d.) of $\sim 0.1 \mathrm{~m}$ for 7 -point averages. AltiKa's $\sigma^{0}$ values are 2.5-3 dB less than those from Jason-2, with a s.d. of $\sim 0.3 \mathrm{~dB}$, with these relatively large mismatches to be expected as AltiKa measures a different part of the spectrum of sea surface roughness. A new wind speed algorithm is developed through matching a histogram of $\sigma^{0}$ values to that for Jason- 2 wind speeds. The algorithm is robust to the use of short durations of data, with a consistency at roughly the $0.1 \mathrm{~m} / \mathrm{s}$ level. Incorporation of $\mathrm{H}_{\mathrm{s}}$ as a secondary input reduces the assessed error at crossovers from $0.82 \mathrm{~m} / \mathrm{s}$ to 0.71 $\mathrm{m} / \mathrm{s}$. A comparison across all altimeter frequencies used to date demonstrates that the lowest wind speeds preferentially develop the shortest scales of roughness.
\end{abstract}

Keywords: AltiKa altimeter, $\mathrm{K}_{\mathrm{a}}$-band, Sigma0, Wind speed, Wave height, Histograms

\section{Introduction}

The SARAL spacecraft launched on 25th February 2013, is the first to carry a radar altimeter operating at $\mathrm{K}_{\mathrm{a}}$-band $(35.75 \mathrm{GHz})$. Its primary mission is to measure the range to Earth, and hence sea surface elevation, but altimeters also provide useful information on wind, waves and rain. To help generate consistent datasets for climate change studies it is important that the calibration of a new instrument is unbiased with respect to preceding and contemporaneous altimeters. In this paper I concentrate principally on the minimization of any differences between AltiKa and other altimeter datasets, rather than on comparisons against non-satellite data. The mission plans for Jason-1 and Jason-2 had tandem phases that allowed more than 6 months of observations simultaneous with their predecessors in that particular orbit. This enabled detailed cross-calibration studies between Jason-1 and TOPEX (Quartly 2004; Tournadre 2004; Tournadre and Bhandari 2009) and then between Jason-1 and Jason-2 (Quartly 2009b, Quartly 2010a; Quartly 2010b). Although AltiKa occupies the track previously surveyed by ERS-1, ERS-2 and Envisat, there was no overlap in time with Envisat as its altimeter stopped working in April 2012. Therefore greater analytical effort is required to achieve a robust merging of AltiKa metocean data with other products.

Since AltiKa operates at a frequency not previously used for altimeters, the challenges are two-fold - firstly improving the quantification and editing of the atmospheric attenuation (which is not covered here), and secondly developing a geophysical model function to relate the normalised backscatter, $\sigma^{0}$, at the $\mathrm{K}_{\mathrm{a}}$-band scale to wind speed at $10 \mathrm{~m}, \mathrm{u}_{10}$. The numerical inversion of waveforms to give significant wave height, $\mathrm{H}_{\mathrm{s}}$, is very well established; however validation of its output is also required. In this paper I examine the comparison of AltiKa data with Jason-2 observations, firstly through a match up of dual satellite crossovers (section 2), and then via a novel technique of equating histograms (section 3). Section 4 includes both a summary of the key points and a discussion of the merits of vicarious calibration against another altimeter. 


\section{Jason-2 -AltiKa crossover analysis}

Direct comparisons of altimeter data with in situ records from buoys suffer from three discrepancies. The first is the essential difference in nature of the properties being compared, with altimeters offering a momentary view over a footprint of $\sim 7 \mathrm{~km}$ diameter whilst the buoy provides typically a 20-minute average of the wave properties at a specific location, with waves under a period of a few seconds not resolved. There will also be errors due to the spatial variation in wave conditions if the altimeter does not fly directly over the buoy, and temporal variations if the overflight does not coincide with the time of the buoy observations (with buoys usually recording average wave conditions only every 1-3 hours to save on battery life).

\subsection{Data sources and match-up requirements}

From autocorrelation analysis along altimeter tracks and through buoy time series, Monaldo (1988) suggested these spatial and temporal errors would be at an acceptable level if the separations are less than $50 \mathrm{~km}$ and 30 minutes respectively. Similar values have been adopted for altimeter validation by other researchers (Cotton and Carter. 1994, Queffeulou 2004), whilst Ray and Beckley (2012) expanded the spatial threshold to $70 \mathrm{~km}$. Matchups between altimeters in orbits of different inclination are likely to result in the tracks crossing one another i.e. minimal spatial separation. However Queffeulou (2003) widens the time constraint to within one hour and applies $100 \mathrm{~km}$ along-track smoothing, whilst Gourrion et al. (2002) in their exhaustive comparison of altimeter and scatterometer matchups used a range of thresholds with the most restrictive being 30 minutes and $15 \mathrm{~km}$.

If the buoy data are taken as ground truth, the errors in wave height for Jason-2 are between $0.23 \mathrm{~m}$ and $0.39 \mathrm{~m}$ depending upon analytical approach (Abdalla et al., 2010; Ray and Beckley, 2012) and $\sim 1.5$ $\mathrm{m} \mathrm{s}^{-1}$ for wind speed (Abdalla et al., 2010). However, given the above discussion on the nature of the causes of some of these errors, the discrepancies between two altimeters might be expected to be smaller. For example, the tandem mission of Jason-1 and Jason-2 showed r.m.s. differences of $0.17 \mathrm{~m}$, for $\mathrm{H}_{\mathrm{s}}$ (Quartly, 2010a), which were taken with negligible time difference, and these reduced considerably when along-track averaging was applied as well. Using along-track averages, Zieger et al. (2009) noted the

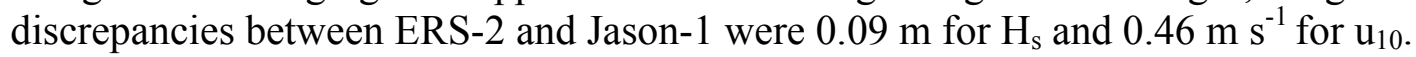

In this work I have examined near-simultaneous ocean observations by AltiKa and Jason-2, which is a conventional (low rate mode) radar altimeter operating during the first years of AltiKa's mission. The data used were the AltiKa GDRs for cycles 1-12 and IGDRs for $13 \& 14$, so that all are version T, having the 'patch 2' corrections for atmospheric attenuation. The corresponding Jason-2 data were the IGDRs for cycles 175 to 221 (version D). In this work I have defined matchups as when the two satellites observe points within $50 \mathrm{~km}$ of each other and within 30 minutes. [Data outside the latitude range $55^{\circ} \mathrm{S}$ to $65^{\circ} \mathrm{N}$ were discarded to avoid any possible contamination by sea-ice. Land contamination was avoided by ensuring that both the altimeter and radiometer terrain flags were set to 'ocean' and that the water depth was greater than $200 \mathrm{~m}$. Furthermore, data were only retained if all the quality control flags for $\mathrm{H}_{\mathrm{s}}$ and $\sigma^{0}$ were set to zero and the standard deviations of the $1 \mathrm{~Hz}$ values were non-zero i.e. there was intrinsic measurement variability.]

In the 456-day period analysed there were 1893 suitable matchups, but they were not uniformly distributed in either space (Fig. 1a) or time (Fig. 1b). The turning latitude of the satellite orbits $\left(66^{\circ}\right.$ for Jason-2, $82^{\circ}$ for AltiKa) means that they spend relatively more time near these latitudes, and the higher relative angle between their tracks increases the likelihood of crossovers at high latitudes (Fig. 1c). This implies that the matchups tend, on average, to occur at higher wind and wave conditions than the global average (not shown).

In fact the peculiarities of the orbits mean that for some 30-day periods all the matchups occur at high latitudes, whilst for subsequent ones they are all at low to moderate latitudes (Fig. 1b). This pattern repeats every 117 days, corresponding to the relative precession of the two orbits (AltiKa being in a sunsynchronous orbit). As most of the matchups correspond to one track crossing another, the separation of nominal 1-second observations is predominantly less than $4 \mathrm{~km}$ (Fig. 1d) with the time separation 
uniformly spread between -30 and 30 minutes (not shown). As well as considering individual $1 \mathrm{~Hz}$ measurements, averages were calculated from the 7 points in the track nearest to the crossover.

\subsection{Statistics of crossover comparisons}

A scatter plot of $1 \mathrm{~Hz} \mathrm{H}$ s estimates from the two altimeters shows a near 1:1 relationship, with few outliers (Fig. 2a), with most of these corresponding to data already flagged as suspect in one or other of the data streams. The mean relationship, calculated in $0.5 \mathrm{~m}$ wide $\mathrm{H}_{\mathrm{s}}$ bins indicates that AltiKa records a value a few centimetres higher on average (Fig. 2c), with the standard deviation (s.d.) of the scatter (an indicator of the measurement error) being $\sim 0.2 \mathrm{~m}$ provided the data quality flags are heeded (Fig. 2e). The use of 7-point averages reduces the s.d. to $\sim 0.1 \mathrm{~m}$, a reduction of $\sim \sqrt{ } 4$, which indicates that a significant part of the disparity between the two measurements is just random noise, but some is due to changes in conditions between the two observation times. These values are smaller than those for matchups of altimeters with buoys (e.g. Queffeulou, 2003; Abdalla et al., 2010; Ray and Beckley, 2012). However, they are similar to those found for inter-altimeter comparisons of Jason-1 with ERS-2 (Zieger et al., 2009) and a little larger than the Jason-2 matchups with Jason-1 during the tandem phase (Quartly, 2010a), when the time difference was negligible.

A comparison of the normalised backscatter, $\sigma^{0}$, observed by the two instruments is more complicated because the observations are at two very different frequencies (Jason-2 operates at $13.6 \mathrm{GHz}$ compared with AltiKa at $35.75 \mathrm{GHz}$ ). Again, aside from a few outliers mostly identified by the quality flags, there is a clear envelope to the comparison (Fig. 2b), with a mean offset of $\sim-2.5 \mathrm{~dB}$ (Fig. 2d) and a s.d. of $0.35 \mathrm{~dB}$ (Fig. 2f). The use of 7-point averages does not markedly reduce the s.d., indicating that the variability is not mainly due to random noise or factors having a short wavelength. For comparison, the corresponding scatter for Jason- $2 \mathrm{~K}_{\mathrm{u}}$ and C-band observations is $\sim 0.10 \mathrm{~dB}$ (Quartly, 2010a) and $\sim 0.18$ $\mathrm{dB}$ for Envisat's $\mathrm{K}_{\mathrm{u}^{-}}$and S-bands (Quartly and Tournadre 2004), but these dual-frequency comparisons come from single spacecraft, so there is no disparity in time or space of the observations.

There are nearly 2000 points fulfilling my matchup criteria within these 15 months of the mission, and these provide a very useful assessment of comparable variables e.g. $\mathrm{H}_{\mathrm{s}}$, and offer a means through a $\mathrm{K}_{\mathrm{u}}$-band equivalence to construct a wind speed algorithm for $\mathrm{o}^{0}$ at $\mathrm{K}_{\mathrm{a}}$-band. However there will still be significant uncertainty at very low and very high wind speeds, which are poorly represented within the matchup dataset. In the next section I propose a technique that uses many orders of magnitude more points to construct a $\mathrm{K}_{\mathrm{a}}$-band wind speed algorithm, and which allows the investigation of temporal variation or $\mathrm{H}_{\mathrm{s}}$ dependency.

\section{Algorithm development through histogram matching}

For TOPEX, Freilich and Challenor (1994) proposed the development of a wind speed algorithm based on matching a histogram of $\sigma^{0}$ observations with the global probability distribution function (p.d.f.) of wind speeds, but maintaining a given functional form for the p.d.f.. Here that restriction is dropped. In essence global p.d.f.s are constructed of the observable and of the desired measurement and the various percentiles in the cumulative distribution function matched up. In this case, it's an inverse relationship, with the 10th, 20th, 30th percentiles of wind speed (Fig. 3a) corresponding to the 90th, 80th, 70th percentiles for $\sigma^{0}$ (Fig. 3b). (In practice the p.d.f.s being compared are not strictly 'global', as the editing and quality-control checks mentioned earlier discard data in the coastal zone and outside the latitude range $55^{\circ} \mathrm{S}$ to $65^{\circ} \mathrm{N}$.)

Such a bravura connection between two different sets of observations cannot be usefully constructed for arbitrary parameters. This technique for linking AltiKa's $\sigma^{0}$ observation to wind speed is facilitated by the strong expectation that there will be a causal relation between the two (as has been observed for $\mathrm{K}_{\mathrm{u}}$-band, C-band and S-band altimetry) and that this will be a monotonic relationship. This equivalence is only practicable because the Jason-2 and AltiKa altimeters sample the same areas of ocean, albeit at different times, with no net change in the global environment between the two sets of measurements. Whilst one instrument may sample a major storm not sensed by the other, the p.d.f. of 
conditions under the Jason-2 tracks and under the AltiKa tracks are expected to be the same. On the other hand, a comparison with a reanalysis field would suffer from the latter being effectively smoothed due to its coarser resolution.

Under these assumptions, a mere 12 days of data, with typically 4000001 -Hz records for Jason-2 and AltiKa, leads to a smooth relationship spanning the majority of $\sigma^{0}$ observations (Fig. 3c). Strictly the two histograms need adjusting to correct for the fact the altimeters have different latitudinal sampling distributions (Fig. 4), with Jason-2's observation density being enhanced near its turning latitude $\left( \pm 66^{\circ} \mathrm{N}\right)$. However, the effect of applying such a change is too small to be shown on Fig. 3c.

\subsection{Temporal variations of comparison}

As there are so many $1 \mathrm{~Hz}$ observations in a few days, this calibration process can be repeated sequentially on short segments of data that are effectively independent of one another. I have performed such an analysis for 38 consecutive 12-day periods, with the anomalies relative to the 15-month mean relationship shown in Fig. 5a. (A period of 12 days was chosen so that both instruments have a roughly even distribution over all ocean basins, however a value too close to Jason-2's repeat period of 9.92 days was avoided to demonstrate that the variability of the relationship is small even for non-uniform global sampling by both instruments.)

The standard deviation (s.d.) of the 38 independently derived relationships (Fig. 5b) serves as an indicator of the error associated with this methodology. In fact there appears to be consistently higher values over days 250-300; however, separate examination of the histograms of AltiKa $\sigma^{0}$ and of Jason-2 wind speeds did not show either to be particularly anomalous. There is also a marked periodic offset (noticeable at high $\sigma^{0}$ ) for roughly every 5 th period. This can be directly connected to $\sigma^{0}$ changes of the Jason-2 instrument, associated with the fraction of time the instrument spends in the shadow of the Earth (Quartly 2010a), which changes as the orbit precesses. Having found this periodicity in the algorithm developed from histogram matching, one can also note its presence within the matchup dataset (not shown).

\subsection{Variation with wave height}

Many of the well-established wind speed algorithms at $\mathrm{K}_{\mathrm{u}}$-band (e.g. Gourrion et al. 2002) include the influence of wave height in the model. This is physically reasonable, because for a given wind speed at $10 \mathrm{~m}$ above the surface, the presence of large waves will act as a further source of sea surface roughness, and consequently lead to lower $\sigma^{0}$ values. Therefore I now separate the data into $\mathrm{H}_{\mathrm{s}}$ bins of $0.2 \mathrm{~m}$ width for both Jason-2 and AltiKa, noting the good agreement between these two satellites (Fig. 2ac), and equate each of these sets of histograms. Figure 5c shows the results as a contour plot to allow detail to be seen within the wide dynamic range. Since there are much fewer observations at low and high $\mathrm{H}_{\mathrm{s}}$, the data for 0-0.6 m have been compiled into one category, and all the observations above $6.0 \mathrm{~m}$ are in $0.6 \mathrm{~m}$ wide bins too.

The pattern revealed is broadly similar to that shown by Gourrion et al. (2002) for $\mathrm{K}_{\mathrm{u}}$-band. For example a wind speed of $5 \mathrm{~m} / \mathrm{s}$ is associated with a $\sigma^{0}$ value of $\sim 11.5 \mathrm{~dB}$ at $\mathrm{H}_{\mathrm{s}}=5.0 \mathrm{~m}$, but with $\sigma^{0}=11.9$ $\mathrm{dB}$ at $\mathrm{H}_{\mathrm{s}}=0.5 \mathrm{~m}$. Using these results to create a look-up table for a 2-parameter wind speed algorithm, one notes that the dependence on $\mathrm{H}_{\mathrm{s}}$ is much weaker than that on $\sigma^{0}$, but as will be shown later it does significantly improve the quality of wind speed retrievals.

\subsection{Evaluation of wind speed algorithms}

This work has developed two wind speed algorithms for AltiKa, one solely based on $\sigma^{0}$ (Fig. 3c), and one using both $\sigma^{0}$ and $\mathrm{H}_{\mathrm{s}}$ (Fig. 5c). In addition there is a wind speed value in the AltiKa data files, which comes from a 1-parameter algorithm developed by Lillibridge et al. (2014) through comparison of AltiKa $\sigma^{0}$ values with collocated wind speeds from the ECMWF reanalysis. I compare the wind speed estimates from these three algorithms with that from the Jason-2 data at the crossover points. Although 
the algorithms developed in this paper have been based on Jason-2 data, there has been no direct use of the near-simultaneous measurements at crossovers, so the algorithms are effectively independent of these specific points.

Figures $6 a, 6 b \& 6 c$ show the scatter plots for these 3 algorithms against Jason- 2 values of $u_{10}$. The points in common to all three datasets are shown by light grey crosses; those that are only valid for some datasets are shown as black circles. At this stage, the 2-parameter algorithm derived in this paper is only defined over the region shown by contours in Fig. 5c i.e. that part of $\sigma^{0}-\mathrm{H}_{\mathrm{s}}$ space for which there are sufficient observations to give a robust retrieval. Consequently no value is returned for this algorithm for combinations of low $\mathrm{H}_{\mathrm{s}}$ and low $\sigma^{0}$, or for $\mathrm{H}_{\mathrm{s}}>3 \mathrm{~m}$ for high $\sigma^{0}$. The comparison statistics for the three algorithms (Figs. 6d \& 6e) are thus computed using only the points valid for all algorithms, with 7-point along-track averages used in all cases for both AltiKa and Jason-2.

Both algorithms developed in this paper return values biased a little lower than Jason-2 does at the crossovers (Fig. 6d), with the r.m.s. scatter being about $0.7 \mathrm{~m} / \mathrm{s}$ (Fig. 6e). The algorithm of Lillibridge et al. (2014) has less overall absolute bias, except for at low wind speeds (which it overestimates) and at high wind speeds (which it underestimates). The r.m.s. error associated with these algorithms is $0.82 \mathrm{~m} / \mathrm{s}$ for my 1-parameter algorithm and $0.72 \mathrm{~m} / \mathrm{s}$ for both the other ones . As the histogram-matching technique developed here utilises Jason-2 records of wind speed, it is able to cover the extremes (both low and high wind speed) better than Lillibridge et al (2014), which is based on a coarser resolution reference field coming from the ECMWF reanalysis. However their algorithm performs slightly better over a wide range of moderate conditions.

Of course comparison with Jason-2 is not the only assessment needed to fully validate a wind speed algorithm. However a strict consistency between altimeter missions is required for long-term climate studies not just of mean wind speed conditions in a region, but also of the occurrence of extremes.

\section{Summary and discussion}

This paper assesses the quality of AltiKa metocean data for merging with those from other altimeters for long-term trend analyses e.g. Young et al. (2011). The estimates of wave height are very good, with a slight overestimate relative to Jason-2 of a few centimetres, and an r.m.s. difference of the 7point averages at crossovers of $\sim 0.1 \mathrm{~m}$. The $\sigma^{0}$ values at $\mathrm{K}_{\mathrm{a}}$-band are several $\mathrm{dB}$ less than at $\mathrm{K}_{\mathrm{u}}$-band, which reflects that they are observing a different part of the spectrum of sea surface roughness

In subsequent analysis I assume that the AltiKa $\sigma^{0}$ values are an accurate estimation of the normalised backscatter at the surface, neglecting the fact that work is ongoing to improve the quality of the atmospheric corrections. Thus I develop a $\mathrm{K}_{\mathrm{a}}$-band wind speed algorithm, simply by equating histograms of $\sigma^{0}$ and an independent measure of wind speed with approximately the same spatial resolution and global distribution. Abdalla (2014) has also developed a $\mathrm{K}_{\mathrm{a}}$-band algorithm through histogram adjustment, but his approach involves maintaining an expected functional form, whereas the one detailed here yields a purely empirical relationship. The approach of Lillibridge et al. (2014) used matchups with a global model. All three of these ideas can generate a candidate wind speed algorithm from only tens of days of data, rather than requiring long time series to acquire sufficient buoy matchups.

By developing my algorithm using Jason-2 data, I have ensured that it is consistent with altimeter records of $\mathrm{u}_{10}$, but it has not necessarily produced an AltiKa dataset that will blend seamlessly with alternative sources of wind data. In particular this is not an absolute calibration, and will be susceptible to any biases within the Jason-2 algorithm. The wind speeds used here came from the Jason-2 4-parameter retracker, which is the one commonly used by most researchers although the 3-parameter one has less spatial variability (Quartly, 2009a) and may be a more trustworthy estimate. However, the use of 7-point averages for the final evaluation (Fig. 6) removes most of the difference between these two fields. Another disadvantage of a Jason-2-based algorithm is that a vestige of the 59-day precession cycle (Quartly, 2010a) is discernible if multiple short periods of data are analysed (Fig. 5a).

The histogram-matching technique was introduced to convert $\mathrm{K}_{\mathrm{a}}$-band $\sigma^{0}$ directly to wind speed rather than to an equivalent $\mathrm{K}_{\mathrm{u}}$-band $\sigma^{0}$, so that the extension to a 2-parameter model was straightforward. 
The effect of $\mathrm{H}_{\mathrm{s}}$ is small but noticeable (Fig. 5c) and reduces the r.m.s. error at the crossover points by $14 \%$ (from $0.82 \mathrm{~m} / \mathrm{s}$ to $0.71 \mathrm{~m} / \mathrm{s}$ ). This developed 2-parameter algorithms does not perform as well as the current AltiKa wind speed algorithm in moderate conditions, but gives a better performance at extremely low and extremely high wind speeds. Presently the new 2-parameter algorithm is not defined for regions of $\sigma^{0}-\mathrm{H}_{\mathrm{s}}$ space that are poorly sampled; some merging with the 1-parameter algorithm could be developed to cover such cases, but it is clear that many of the large errors of Lillibridge et al. (2014) are associated with such conditions. One issue not fully addressed as yet is that of the quality control of the data. Here all standard flags have been heeded, but it is noted that these discard any AltiKa $\sigma^{0}$ values below $5 \mathrm{~dB}$, which potentially affects the ability to construct an algorithm covering the very highest wind speeds.

Finally, the development of a 1-parameter $\mathrm{K}_{\mathrm{a}}$-band algorithm consistent with those from other altimeters at different frequencies allows some reflection on the changes in sea surface roughness at different scales as wind speed increases. Figure $7 \mathrm{a}$ shows the backscatter strength at nadir for the 4 frequencies that have been used in altimetry - although the absolute calibration for some of these frequencies is unclear, their shapes show a common theme, with different slopes for the asymptotes. Figure $7 \mathrm{~b}$ emphasises the differences by comparing $\mathrm{K}_{\mathrm{a}}, \mathrm{C}$ and $\mathrm{S}$ to $\mathrm{K}_{\mathrm{u}}$, subtracting the lower frequency (longer wavelength) from the other, noting that the magnitudes may be erroneous because of uncertainties in the absolute calibration of the different frequencies. These shapes have been well documented for $\mathrm{K}_{\mathrm{u}^{-}}$ C (Topex, see Quartly et al 1996) and $\mathrm{K}_{\mathrm{u}} \mathrm{S}$ (Envisat, see Quartly and Tournadre 2004) although normally as a function of $\sigma^{0}$. As wind speed increases, sea surface roughness increases first at the smallest scales, and then at larger wind speeds the contribution for larger surface wavelengths increases more quickly; Figure $7 \mathrm{~b}$ provides quantification of this concept. The relationship between different frequencies at the lowest wind speeds may be hard to assess, because under these conditions there may often be inhomogeneities within the altimeter footprint, manifesting themselves as "bright targets" (Gómez-Enrí et al., 2010). Also at the shortest scales, which are those sensed at $K_{a}$-band, the surface physics are dominated more by surface tension than gravity that controls the larger scales.

\section{Acknowledgements}

This work was carried out as part of the KaNUTE project, and was supported through the PML research programme Altika - Innovative Meteorological Studies.

\section{References}

Abdalla, S., P.A.E.M. Janssen, and J.-R. Bidot, 2010. Jason-2 OGDR wind and wave products: monitoring, validation and assimilation. Marine Geodesy 33, 239-255.

Abdalla, S., 2014. Calibration of SARAL/AltiKa wind speed. IEEE Geoscience and Remote Sensing Letters, 11 (6), 1121-1123. doi: 10.1109/LGRS.2013.2287805

Cotton, P.D., and D.J.T. Carter, 1994. Cross calibration of TOPEX, ERS-I, and Geosat wave heights. Journal Geophysical Research, 99(C12), 25025-25033, doi:10.1029/94JC02131.

Freilich, M.H., and P.G. Challenor, 1994. A new approach for determining fully empirical altimeter wind speed model functions. Journal Geophysical Research, 99, 25,051-25,062, 1994.

Gómez-Enrí, J., S. Vignudelli, G.D. Quartly, C.P. Gommenginger, P. Cipollini, P.G. Challenor and J. Benveniste, 2010. Modeling Envisat RA-2 waveforms in the coastal zone: Case-study of calm water contamination, IEEE Geoscience and Remote Sensing Letters, 7 (3), 474-478. doi: 10.1109/LGRS.2009.2039193

Gourrion, J., D. Vandemark, S. Bailey, B. Chapron, G.P. Gommenginger, P.G. Challenor, M.A. Srokosz, 2002. A two-parameter wind speed algorithm for Ku-band altimeters. Journal Atmospheric Oceanic Technology, 19, 2030-2048. doi: 10.1175/1520-0426(2002)019<2030:ATPWSA>2.0.CO;2

Lillibridge, J., R. Scharroo, S. Abdalla, and D. Vandemark, 2014. One- and two-dimensional wind speed models for Ka-band altimetry. Journal Atmospheric Oceanic Technology. 31, 630-638.

Monaldo, F., 1988. Expected differences between buoy and radar altimeter estimates of wind speed and significant wave height and their Implications on buoy-altimeter comparisons. Journal Geophysical Research, 93, 2285-2302. 
Quartly, G.D., T.H. Guymer, and M.A. Srokosz, 1996. The effects of rain on Topex radar altimeter data, Journal Atmospheric Oceanic Technology, 13, 1209-1229. doi: 10.1175/15200426(1996)013<1209:TEOROT>2.0. CO;2

Quartly, G.D., 2004. Sea state and rain: A second take on dual-frequency altimetry, Marine Geodesy, 27(1-2), 133-152, doi: 10.1080/01490410490465472. (Errata, Marine Geodesy, 27(3-4), 789-795, doi: $10.1080 / 01490410490905013$ )

Quartly, G.D., 2009a. Optimizing $\sigma^{0}$ information from the Jason-2 altimeter, IEEE Geoscience Remote Sensing Letters, 6, 398-402, doi: 10.1109/ LGRS.2009.2013973

Quartly, G.D., 2009b. Improving the intercalibration of $\sigma^{0}$ values for the Jason-1 and Jason-2 altimeters. IEEE Geoscience Remote Sensing Letters, 6 (3), 538-542. doi: 10.1109/LGRS.2009.2020921

Quartly, G.D., 2010a. Jason-1 / Jason-2 metocean comparisons and monitoring. Marine Geodesy 33 (1), 256-271. doi: 10.1080/01490419.2010.487794

Quartly, G.D., 2010b. Improving the altimetric rain record from Jason-1 \& Jason-2. Journal Geophysical Research. 115, art. no. C03008 (15pp.). doi: 10.1029/2009JC005670

Quartly, G.D., and J. Tournadre, 2004. Defining a rain flag for the Envisat altimeter, Report to ESA's RA2/MWR Cross-calibration and Validation Team. Available from European Space Agency.

Queffeulou, P., 2003. Long-term quality status of wave height and wind speed measurements from satellite altimeters, Proc. of 13th International Society of Offshore and Polar Engineers Conference, 25-30 May, Honolulu, Hawaii, USA .

Queffeulou, P., 2004. Long-term validation of wave height measurements from altimeters. Marine Geodesy, 27 (3-4), 495-510.

Ray, R.D., and B. Beckley, 2012. Calibration of ocean wave measurements by the TOPEX, Jason-1, and Jason-2 satellites, Marine Geodesy, 35:sup1, 238-257. doi: 10.1080/01490419.2012.71861

Tournadre, J. 2004. Validation of Jason and Envisat altimeter dual-frequency rain flags. Marine Geodesy 27, 153-169.

Tournadre, J., and S. Bhandari, 2009. Analysis of short space-time-scale variability of oceanic rain using TOPEX/Jason, Journal Atmospheric Oceanic Technology, 26, 74-90, doi: 10.1175/2008JTECHO605.1.

Young, I.R., S. Zieger, and A.V. Babanin, 2011. Global trends in wind speed and wave height, Science 332, 451-455. doi: 10.1126/science.1197219

Zieger, S., J. Vinoth, and I.R. Young, 2009. Joint Calibration of multiplatform altimeter measurements of wind speed and wave height over the past 20 years, Journal Atmospheric Oceanic Technology, 26, 2549-2564, doi: 10.1175/2009JTECHA1303.1 
Figures
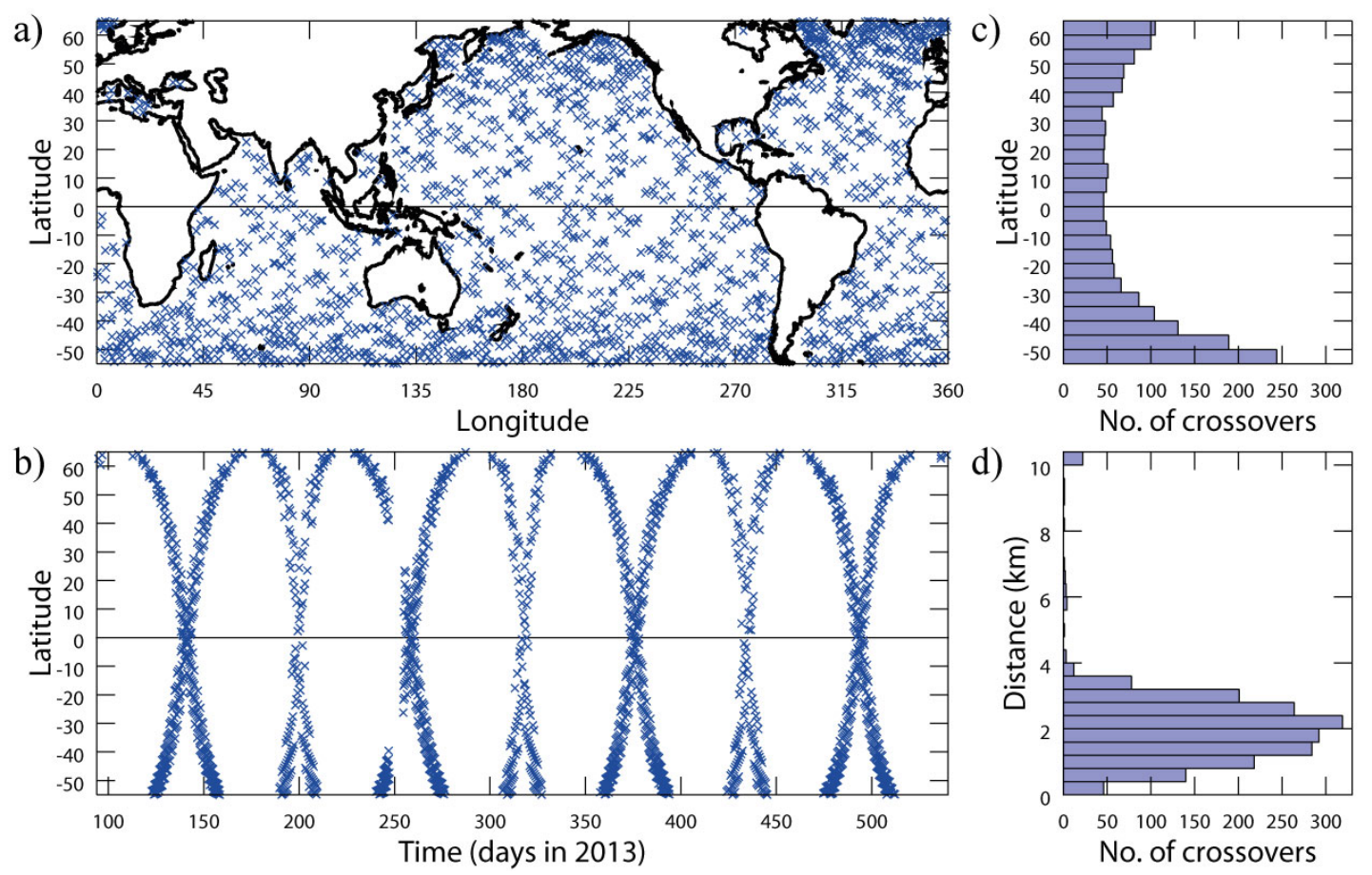

Figure 1. Crossovers between AltiKa and Jason-2 altimeters. a) Locations. b) Latitude as a function of time. c) Histogram of latitude. d) Histogram of distance of separation
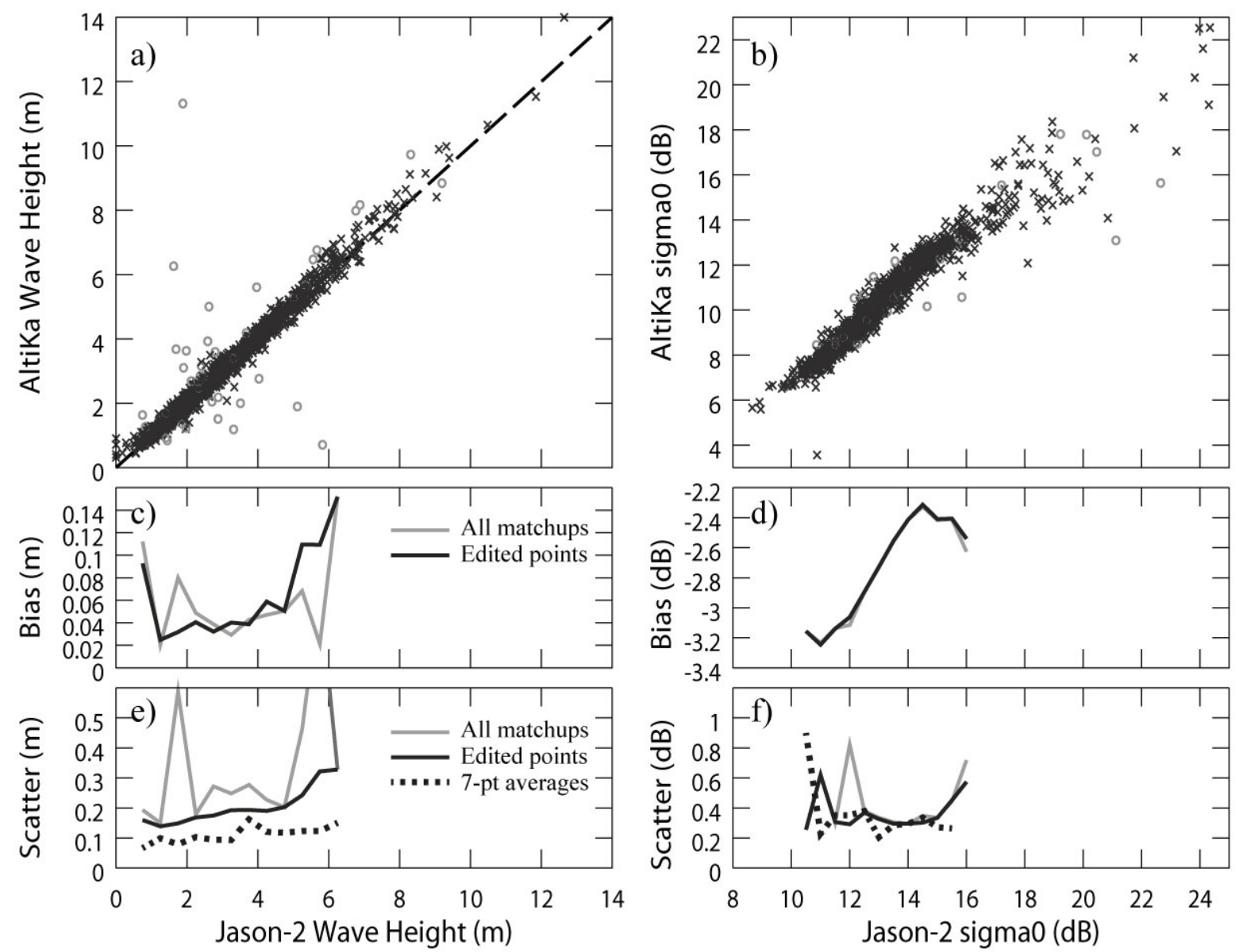

Figure 2. a) Comparison of $1 \mathrm{~Hz} \mathrm{H} \mathrm{H}_{\mathrm{s}}$ values from the 2 altimeters, with ' $\mathrm{x}$ ' for those passing standard quality control tests, and 'o' for those failing. b) Same for $\sigma^{0}$. c) AltiKa mean value of $\mathrm{H}_{\mathrm{s}}$ relative to that of Jason-2. d) Same for $\sigma^{0}$. e) Standard deviation of scatter about mean for $H_{s}$. f) Same for $\sigma^{0}$. Note use of 7-pt average makes a significant reduction to scatter for $\mathrm{H}_{\mathrm{s}}$ but not for $\sigma^{0}$. 
a)

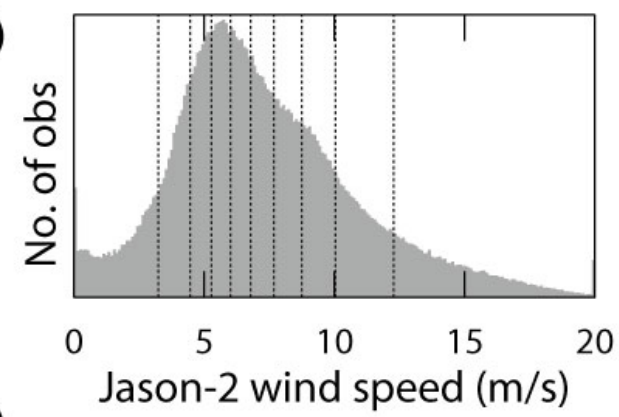

b)

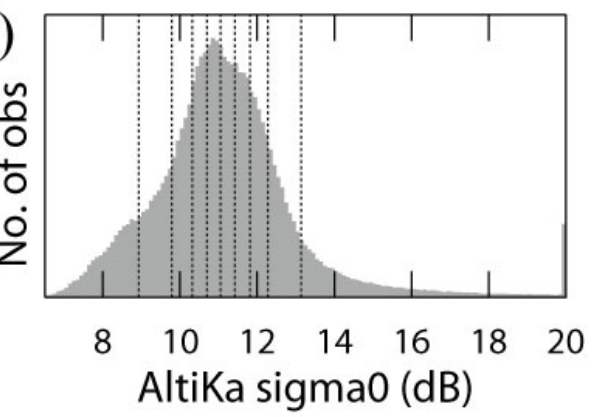

c)

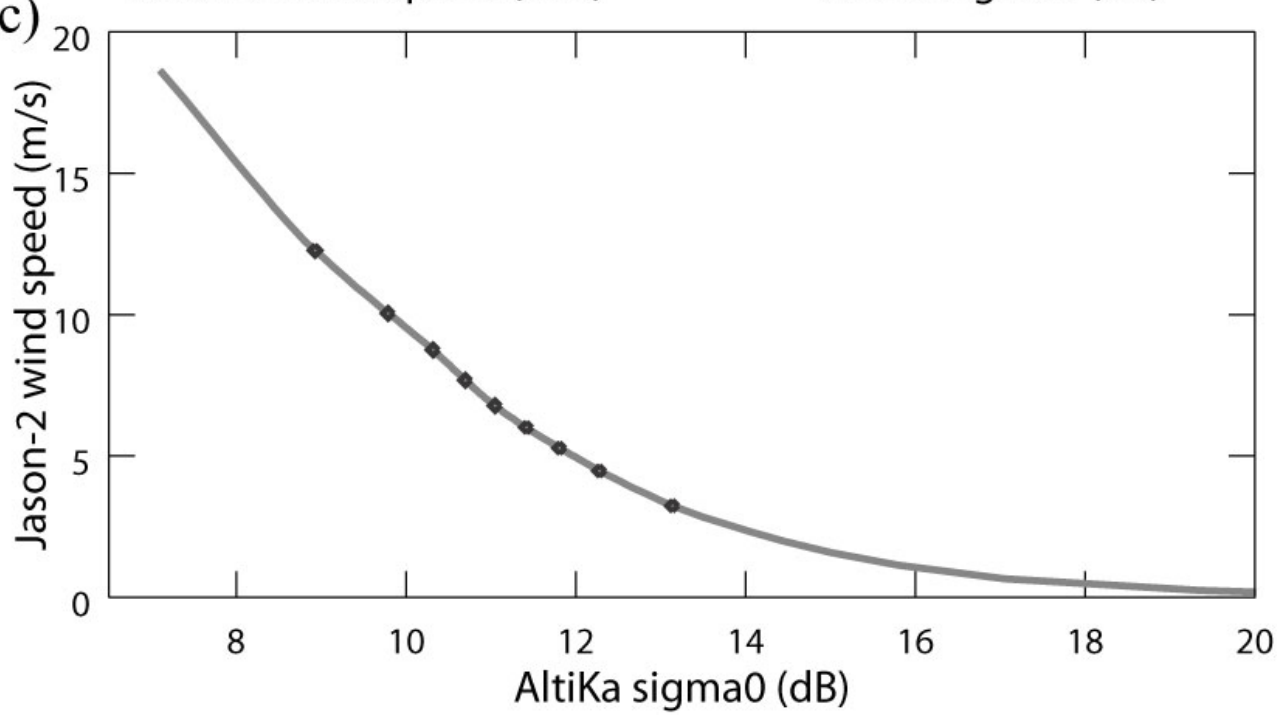

Figure 3. Illustration of histogram-matching technique for a chosen 12-day period. a) Histogram of Jason2 wind speeds, with 10th, 20th, 30th ... 90th percentiles indicated by vertical dotted lines b) Same for AltiKa $\sigma^{0}$ values. c) Match up of percentiles in reverse order from $0.5 \%$ to $99.5 \%$ with the 10 th- 90 th highlighted with circles.

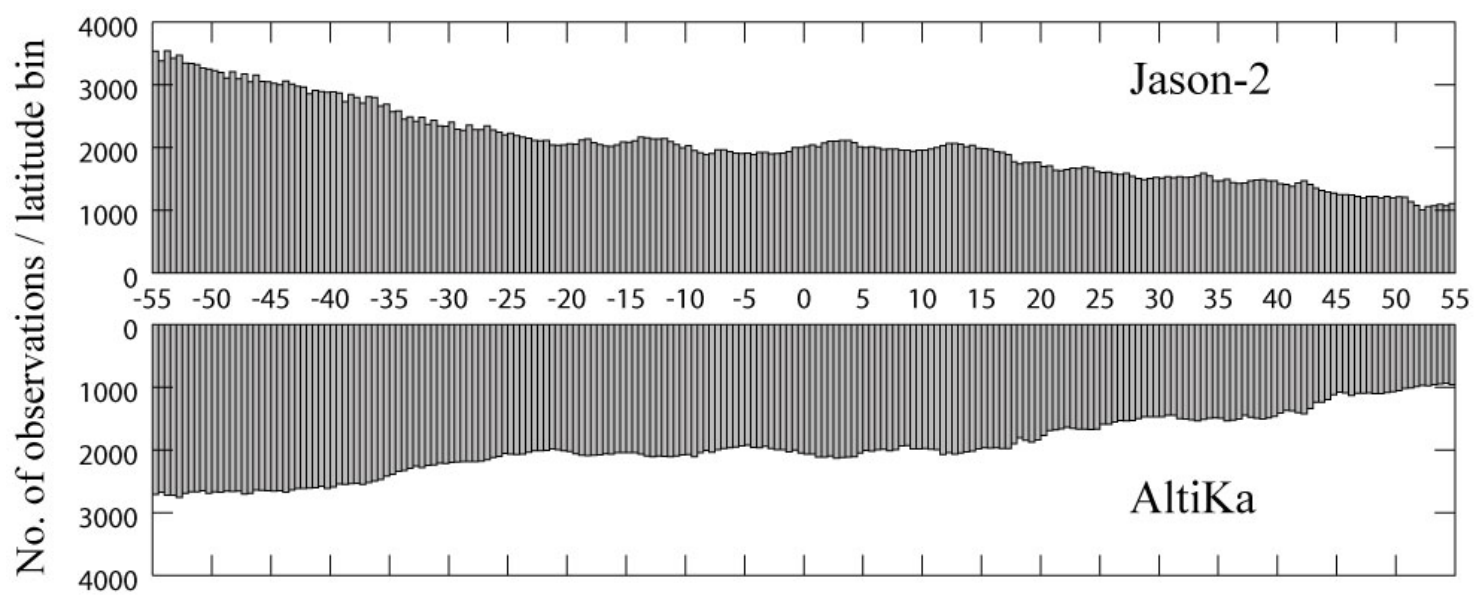

Figure 4. Histograms of Jason-2 and AltiKa observations in $0.5^{\circ}$ wide latitudinal bands, showing that Jason-2 makes relatively more measurements at high latitudes (near its turning latitude). 


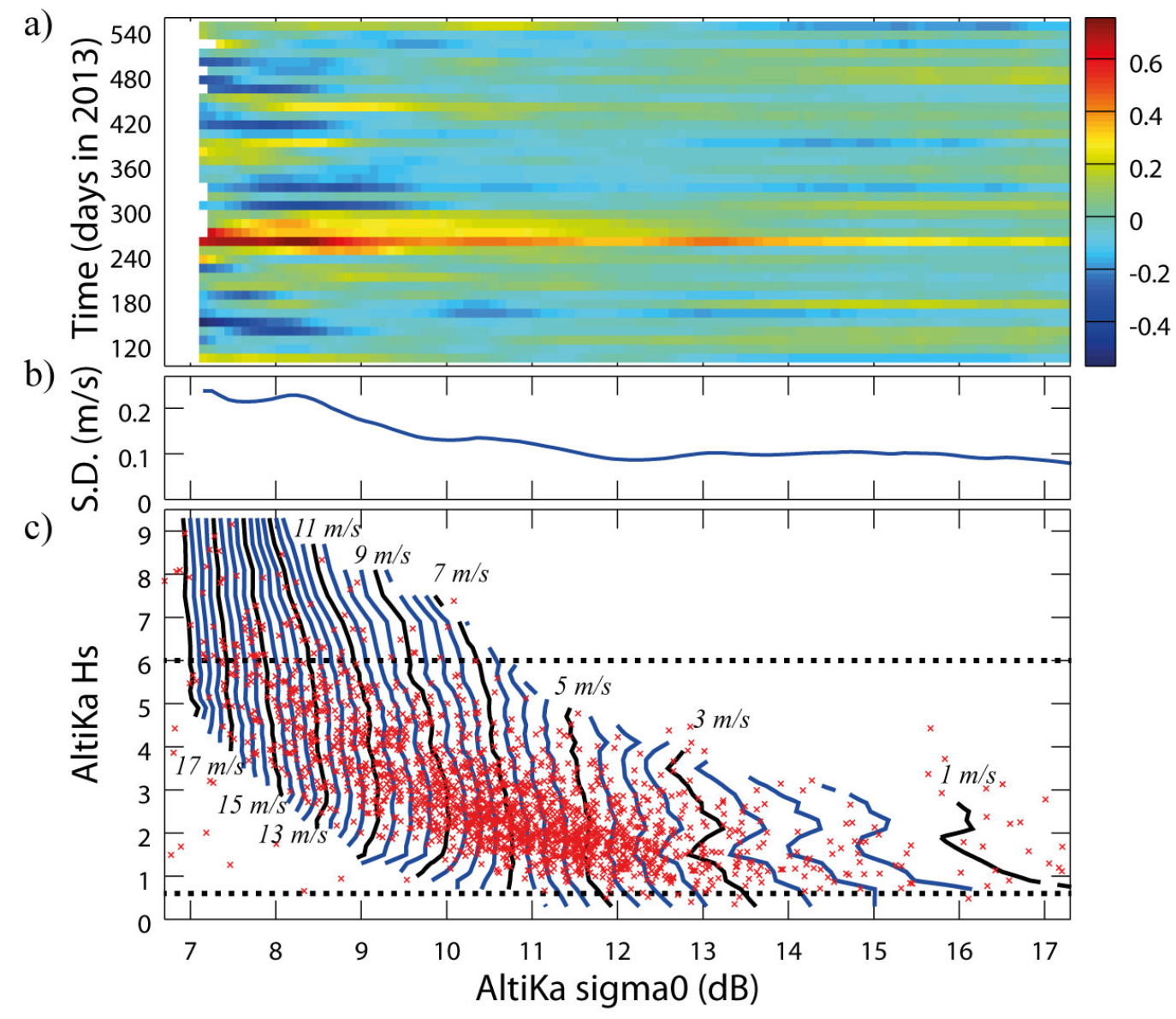

Figure 5. Variations in wind speed algorithm. a) Evaluated separately for each 12-day period, with plot showing anomalies (in $\mathrm{m} / \mathrm{s}$ ) relative to 15 -month mean. b) Standard deviation of these anomalies, as an indicator of the uncertainty in the 1-parameter algorithm. c) Wind speed algorithm evaluated separately for narrow bands of $\mathrm{H}_{\mathrm{s}}\left(0.2 \mathrm{~m}\right.$ in main body, with $0.6 \mathrm{~m}$ wide bins for $\mathrm{H}_{\mathrm{s}}<0.6 \mathrm{~m}$ or $\left.\mathrm{H}_{\mathrm{s}}>6.0 \mathrm{~m}\right)$. Contours are every $0.5 \mathrm{~m} / \mathrm{s}$, with every fourth (in black) labelled. This analysis using global data for the whole of the mission is used to populate a look-up table for the 2-parameter wind speed algorithm. The $\sigma^{0}-H_{s}$ conditions at the matchup points used in the evaluation are indicated by the red crosses. 

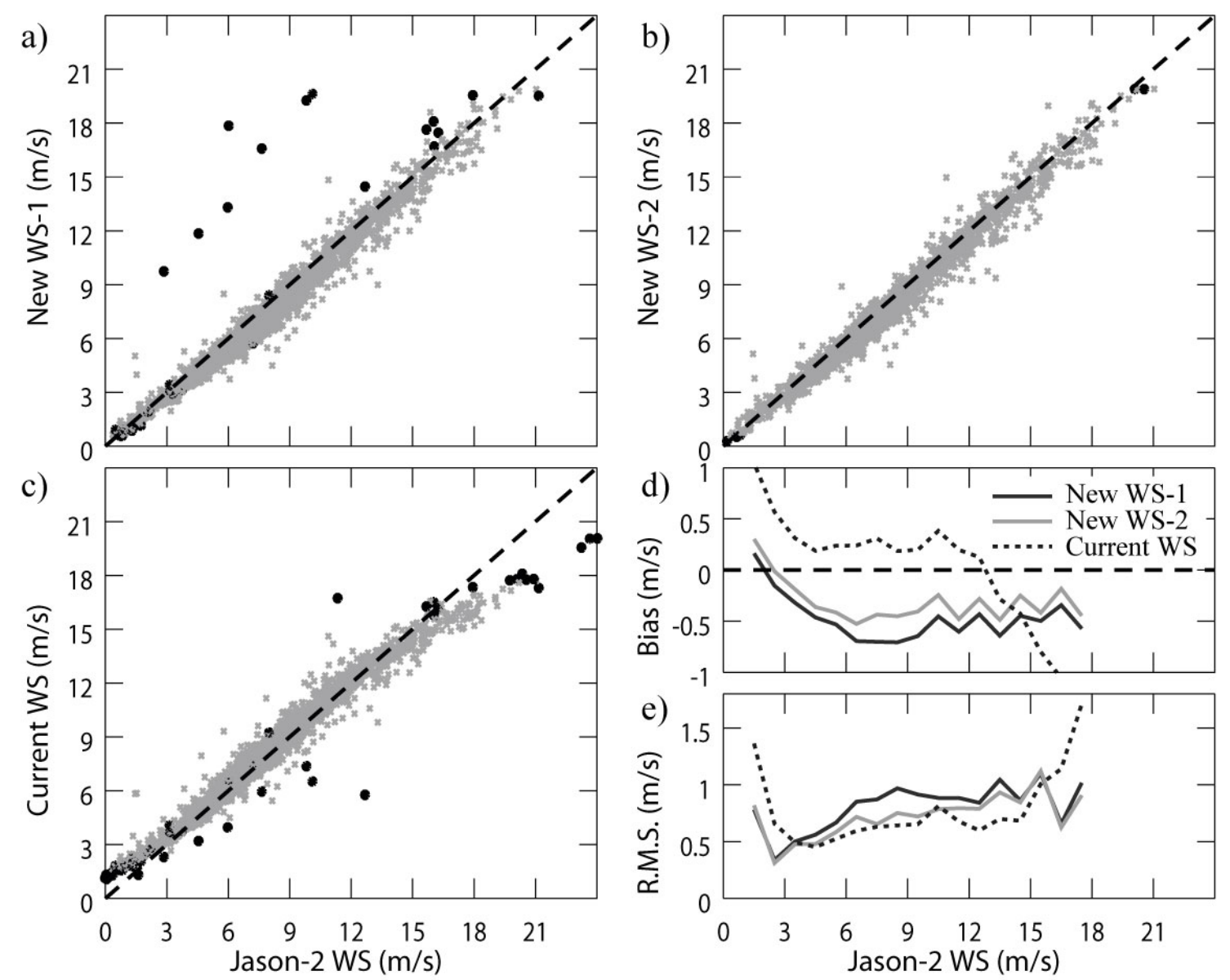

Figure 6. Comparison of 3 AltiKa-based wind speed algorithms against Jason-2 at crossovers. The new 2-parameter algorithm is not defined outside the region indicated in Fig. 5c. Points that are valid for all 3 algorithms are indicated by light grey crosses, whereas those lacking an estimate for one of the other algorithms are shown as black circles. a) 1-parameter algorithm derived in this work. b) 2-parameter algorithm derived in this work. c) Current algorithm detailed by Lillibridge et al. (2014). d) Mean, and. e) R.m.s. of AltiKa estimates relative to Jason-2.
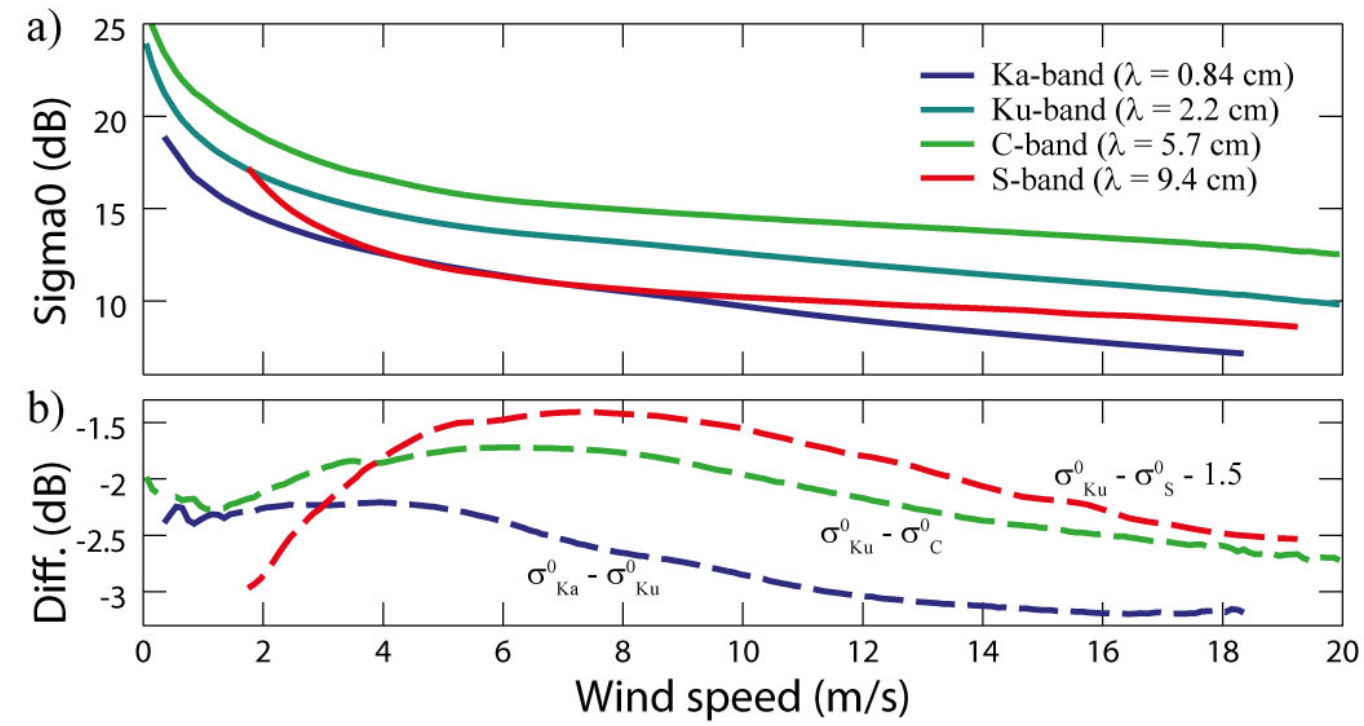

Figure 7. a) Comparison of simple 1-parameter relationships between wind speed and sigma0 at different frequencies $\left(\mathrm{K}_{\mathrm{a}}\right.$ from AltiKa, $\mathrm{K}_{\mathrm{u}}$ and $\mathrm{C}$ from Jason-2, $\mathrm{S}$ from Envisat). Altimeters interact with the sea surface roughness typically at scales of 3 times the radar wavelength and above. b) Difference in $\sigma^{0}$ values relative to $\mathrm{K}_{\mathrm{u}}$-band.. For S-band it is the difference between the two sets of measurements by Envisat, with a $1.5 \mathrm{~dB}$ shift for ease of plotting. 